\title{
Design of Fluorescence-Enhanced Silver Nanoisland Chips for High-Throughput and Rapid Arsenite Assay
}

Hung-Chi Yen, ${ }^{1}$ Tsung-Rong Kuo, ${ }^{* 2,3}$ Min-Hui Huang, ${ }^{1}$ Hao-Kang Huang, ${ }^{1}$ and Chia-Chun Chen $^{*}, 1$

${ }^{1}$ Department of Chemistry, National Taiwan Normal University, Taipei 116 , Taiwan, ${ }^{2}$ Graduate Institute of Nanomedicine and Medical Engineering, Taipei Medical University, Taipei 110, Taiwan, ${ }^{3}$ International Ph.D. Program in Biomedical Engineering, College of Biomedical Engineering, Taipei Medical University, Taipei 110, Taiwan 

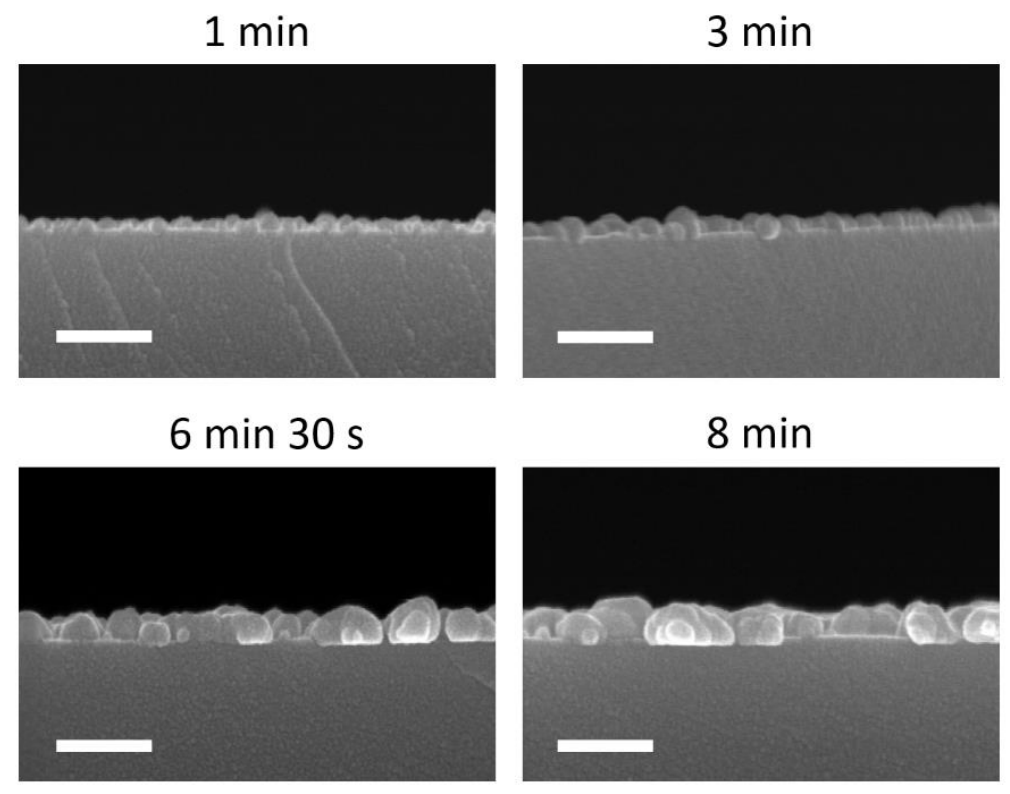

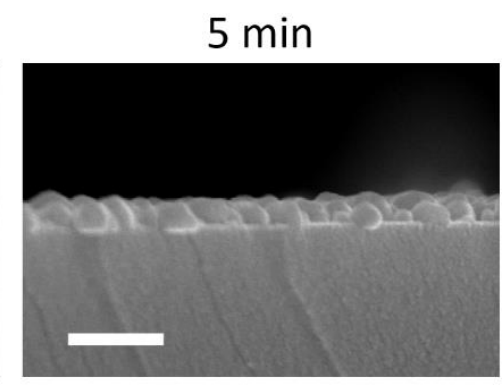

$10 \min$

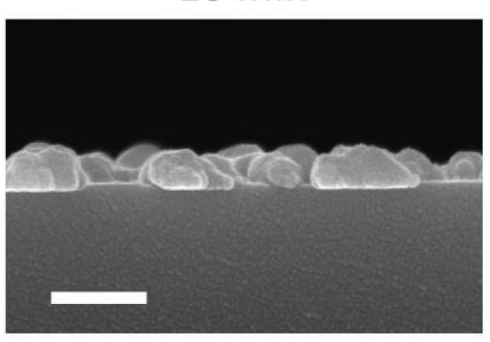

Figure S1. Cross-sectional SEM images of Ag-NIFs with increased growth time from 1 to $10 \mathrm{~min}$. Scale bar: $200 \mathrm{~nm}$.

(a)

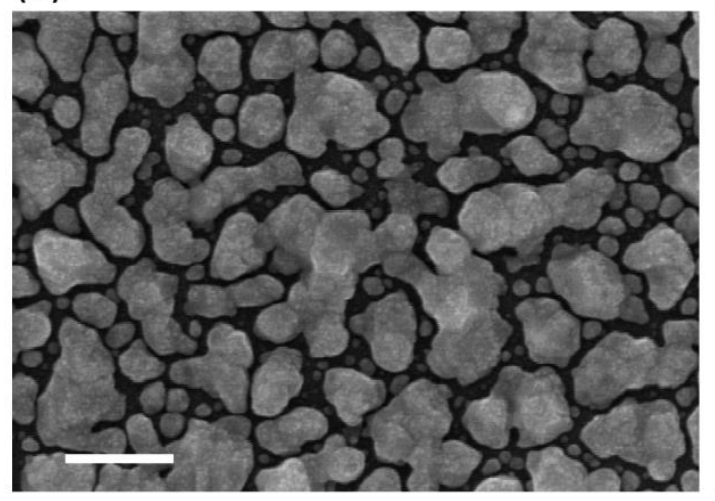

(b)

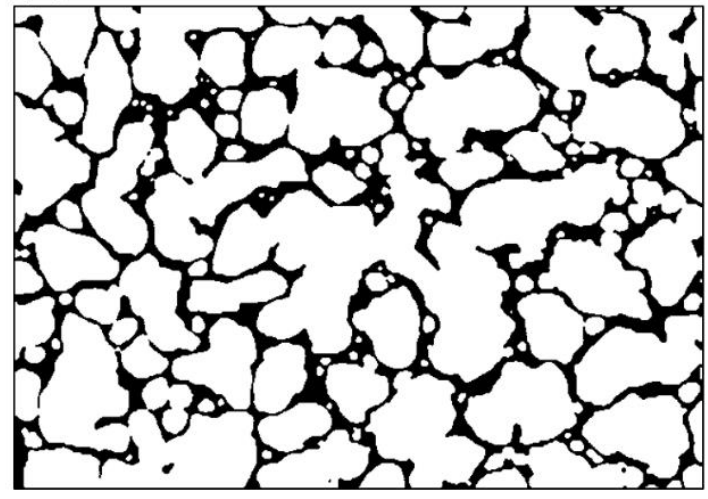

Figure S2. Estimation of the gap area of Ag-NIF by SEM. (a) The original SEM image of the Ag-

NIF grown for 6 min $30 \mathrm{~s}$. Scale bar: $200 \mathrm{~nm}$. (b) The corresponding binary image of the Ag-NIF converted by ImageJ software. 
(a)

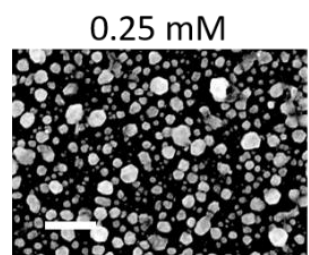

$0.625 \mathrm{mM}$

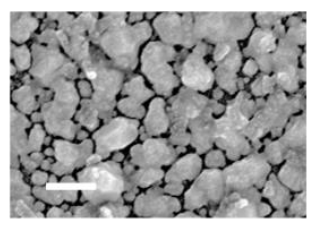

$0.375 \mathrm{mM}$

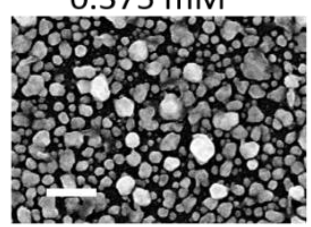

$0.75 \mathrm{mM}$

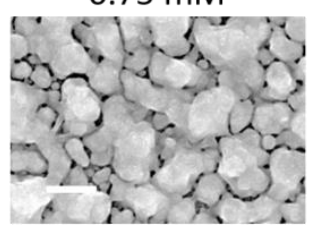

(b)

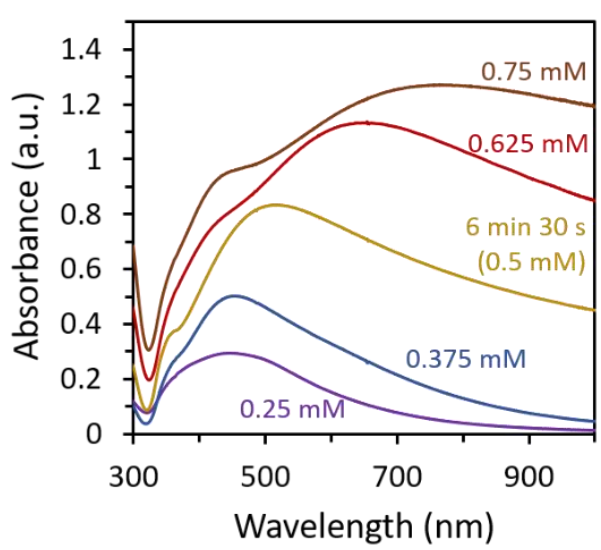

Figure S3. (a) SEM images and (b) UV-vis absorbance spectra of Ag-NIFs with increased concentration of $\mathrm{AgNO}_{3}$ from $0.25 \mathrm{mM}$ to $0.75 \mathrm{mM}$. Scale bar: $200 \mathrm{~nm}$.

(a)

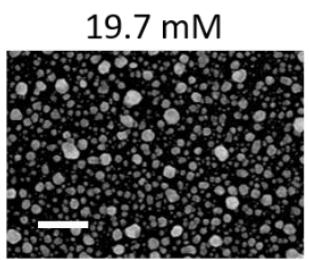

$117.8 \mathrm{mM}$

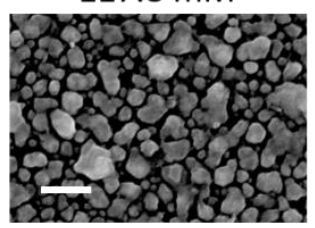

$78.5 \mathrm{mM}$

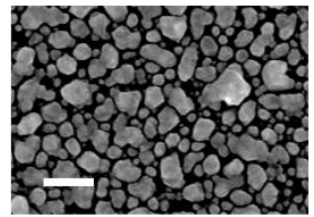

$157.0 \mathrm{mM}$

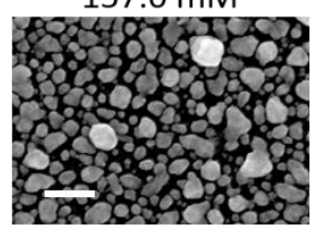

(b)

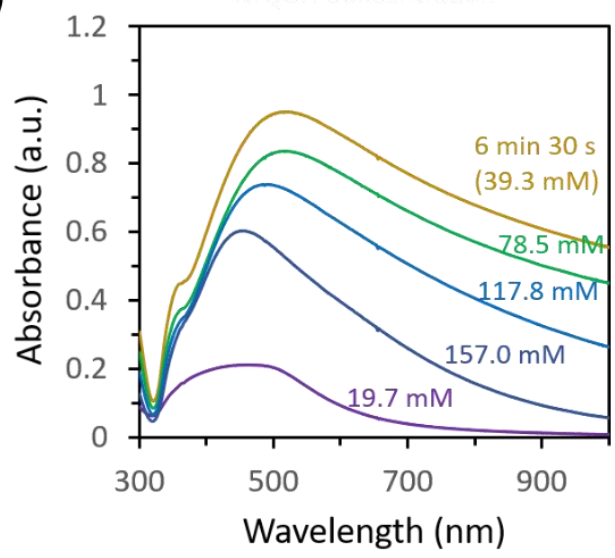

Figure S4. (a) SEM images and (b) UV-vis absorbance spectra of Ag-NIFs with increased concentration of $\mathrm{NH}_{4} \mathrm{OH}$ from $19.7 \mathrm{mM}$ to $157.0 \mathrm{mM}$. Scale bar: $200 \mathrm{~nm}$. 